Oncogenic Mutations in Eutopic Endometrium

\title{
Oncogenic Mutations in Histologically Normal Endometrium: The New Normal?
}

Vivian Lac ${ }^{1}$, Tayyebeh M. Nazeran ${ }^{1,2}$, Basile Tessier-Cloutier ${ }^{3}$, Rosalia Aguirre-Hernandez ${ }^{4}$, Arianne Albert ${ }^{5}$, Amy Lum ${ }^{1}$, Jaswinder Khattra ${ }^{4}$, Teresa Praetorius ${ }^{6}$, Madeline Mason ${ }^{1}$, Derek Chiu $^{1}$, Martin Köbel ${ }^{7}$, Paul J. Yong, ${ }^{8,9}$, Blake C. Gilks ${ }^{2,3}$, Michael S. Anglesio, ${ }^{3,8, *}$, David G. Huntsman ${ }^{1,3,4,8, *}$

\section{Affiliations:}

1. Department of Molecular Oncology, BC Cancer Research Centre, Room 3-218, 675 West 10th Ave, Vancouver, BC V5Z 1L3, Canada

2. Department of Anatomical Pathology, Vancouver General Hospital, 899 W 12th Ave, Vancouver, BC V5Z 1M9, Canada

3. Department of Pathology and Laboratory Medicine, Rm. G227 - 2211 Wesbrook Mall, University of British Columbia, Vancouver, BC V6T 2B5, Canada

4. Contextual Genomics, 2389 Health Sciences Mall \#204, Vancouver, BC V6T 1Z3, Canada

5. Women's Health Research Institute, 4500 Oak St, Vancouver, BC V6H 2N9, Canada

6. Department of Women's Health, Tuebingen University Hospital, Calwerstrasse 7, 72076 Tuebingen, Germany

7. Department of Pathology and Laboratory Medicine, University of Calgary, 2500 University Dr. NW, Calgary, AB T2N 1N4, Canada

8. Department of Obstetrics and Gynaecology, University of British Columbia, Suite 930, 1125 Howe Street, Vancouver, BC V6Z 2K8, Canada

9. BC Women's Centre for Pelvic Pain \& Endometriosis, BC Women's Hospital and Health Centre, Women' Health Centre, F2 - 4500 Oak St., Vancouver, BC V6H 3N1, Canada

\section{Corresponding Authors:}

David G. Huntsman, Department of Molecular Oncology, BC Cancer Research Centre, 675 West 10th Ave, Vancouver, BC V5Z 1L3, Canada. Phone: 604-675-8211, Email: dhuntsma@bccancer.bc.ca

Michael S. Anglesio, Department of Molecular Oncology, BC Cancer Research Centre, 675 West 10th Ave, Vancouver, BC V5Z 1L3, Canada. Phone: 604-675-8211, E-mail: m.anglesio@ubc.ca

\section{Conflicts of Interest:}

DGH is a co-founder and shareholder of Contextual Genomics Inc., a for profit company that provides genomic diagnostics and reporting to assist in cancer patient treatment. RA-H and JK have a patent MOLECULAR QUALITY ASSURANCE METHODS FOR USE IN SEQUENCING pending and are currently employed by Contextual Genomics Inc. The remaining authors have no conflicts of interest to declare. 
Oncogenic Mutations in Eutopic Endometrium

\begin{abstract}
:
The presence of somatic driver mutations in endometriosis has previously been believed to represent early events in transformation, however our group and others have described such mutations in roughly one-third of cases of deep infiltrating or iatrogenic endometriosis. These forms of endometriosis rarely progress to malignancy. Recent studies have also shown somatic driver mutations in normal skin, blood, peritoneal washings, and esophageal epithelium. Such findings prompt speculation on whether such mutations exist in the eutopic endometrium - the likely tissue of origin of endometriosis. In the current study we investigated the presence of somatic driver mutations in histologically normal endometrium from women lacking evidence of gynecologic malignancy or endometrial hyperplasia. Twenty-five women who underwent hysterectomies and 85 women who underwent endometrial biopsies were included in this study. Formalin-fixed, paraffin-embedded tissue specimens were analyzed by means of targeted sequencing followed by orthogonal validation with droplet digital PCR. PTEN and ARID1A immunohistochemistry (IHC) was also performed as surrogates for inactivating mutations in the respective genes. Overall, we observed somatic driver-like events in over $50 \%$ of histologically normal endometrial samples analyzed, which included hotspot mutations in KRAS, PIK3CA, and FGFR2 as well as PTEN-loss by IHC. Analysis of anterior and posterior samplings collected from women who underwent hysterectomies is consistent with the presence of somatic driver mutations within clonal pockets spread throughout the uterus. The prevalence of such oncogenic mutations also increased with age (OR: 1.05 (95\% CI: $1.00-1.10), p=0.035)$. These findings have implications on our understanding of aging and so-called "normal tissues", thereby necessitating caution in the utilization of mutation-based early detection tools for endometrial or other cancers.
\end{abstract}

Keywords: somatic mutations; normal tissue; aging; endometrium; digital PCR 
Oncogenic Mutations in Eutopic Endometrium

\section{Introduction:}

Endometriosis is a chronic disease affecting approximately 10 percent of reproductive-aged women. It leads to a variety of clinical symptoms including chronic (often cyclical) pain, dyspareunia, dysmenorrhea, and infertility[1,2]. Endometriosis is the major risk factor for clear cell and endometrioid carcinomas of the ovary (endometriosis-associated ovarian cancers; EAOCs) [3-5]. Observations of endometriotic lesions (consisting of endometrial-like glands and stroma) contiguous with EAOCs combined with molecular findings from several sequencing studies have further established endometriosis as the precursor of EAOCs[6-8]. In these studies, oncogenic mutations in genes such as ARIDIA and PIK3CA[7,8] have been traced back to endometriotic lesions within the same patients, thereby implicating such alterations as early events towards malignant transformation. However, we have found somatic driver mutations in approximately one-third of deep infiltrating endometriosis and iatrogenic endometriosis cases (forms of endometriosis with little malignant potential)[9,10]. These findings suggest that such mutations may be an inherent feature of endometriosis itself, perhaps conferring survival advantages that allow lesions to persist ectopically. However, in order to study this hypothesis, it is important to clarify whether uterine epithelial mutations can pre-exist endometriosis and be present in the cells prior to implantation at an ectopic site.

In the current study, we sought to explore the mutational landscape of the eutopic (uterine) endometrium from women lacking evidence of gynecologic malignancy by means of targeted sequencing and immunohistochemistry (IHC) studies. Although the origins of endometriosis remain contentious, the concept of retrograde menstruation stands as a leading theory. Herein, endometrial fragments (originating from the uterus) are refluxed upwards through the fallopian tubes and are implanted within the pelvic cavity[11]. This theory is supported by several lines of evidence including the high prevalence of endometriosis in females with congenital outflow obstruction[12] and the successful generation of endometriotic lesions in primate models of endometriosis via intrapelvic injection of menstrual endometrium[13] or cervical occlusion[14]. Considering the dynamic nature of the endometrium as it undergoes many cycles of regeneration and tissue breakdown throughout a woman's reproductive years[15] as well as the recent description of somatic mutations in normal tissues including the skin[16], blood[17], peritoneal washings[18], and the esophageal epithelium[19], it is plausible that somatic mutations may preexist in the eutopic endometrium. Moreover, a series of previous studies have described "latent precancers" (alterations in otherwise normal tissue with unknown contribution to long-term cancer risk) in the form of PTEN-null and PAX2-null glands within histologically normal endometrium[20-22]. Application of next-generation sequencing techniques will broaden our understanding of latent precancers, or pre-existing somatic alterations, in the eutopic endometrium. This is particularly important since these tissues often serves as a reference for molecular studies on endometriosis, endometrial cancer, and EAOCs. 
Oncogenic Mutations in Eutopic Endometrium

\section{Materials and Methods: \\ Patient Identification and Specimen Collection}

We obtained formalin-fixed and paraffin-embedded (FFPE) endometrial tissue specimens from 25 women who underwent hysterectomies (Supplementary Table S1) and 85 women who underwent either dilation and curettage (D\&C) or endometrial biopsies (Supplementary Table S2). As confirmed by pathologist review, all women lacked evidence of gynecologic malignancy or endometrial hyperplasia and all tissue specimens were obtained from the Department of Anatomical Pathology at the Vancouver General Hospital in Vancouver, Canada. For hysterectomy patients (Hx cohort), we collected and subsequently analyzed two blocks to represent endometrial tissue from the posterior uterus and anterior uterus from each patient. For D\&C or endometrial biopsy patients (Bx cohort), we targeted collection of endometrial tissue specimens to represent women of various ages (ranging from 20-61 years of age). Specimen, data collection and experiments were approved by the UBC BC Cancer Agency Research Ethics Board [H05-60119, H02-61375 and H08-01411].

\section{Sample Processing and DNA Extraction}

Hysterectomy specimens were sectioned at $8 \mu \mathrm{m}$ onto glass slides, deparaffinized with xylene and stained with $10 \%$ diluted hematoxylin and eosin (H\&E). Specimens were manually macrodissected to enrich the epithelial layer using the tip of a 20-guage needle under a stereo microscope. DNA from hysterectomy specimens was extracted using the ARCTURUS $®$ PicoPure ${ }^{\circledR}$ DNA Extraction Kit (ThermoFisher Scientific, USA). D\&C and endometrial biopsy specimens were cut into $3-610 \mu \mathrm{m}$ tissue scrolls. DNA from D\&C and endometrial biopsy specimens was extracted using the QIAamp® DNA FFPE Tissue Kit (QIAGEN, USA). All DNA was quantitated using the Qubit 2.0 Fluorometer (ThermoFisher Scientific, USA).

\section{Targeted Sequencing}

All specimens were sequenced using FIND IT $^{\mathrm{TM}}$ version 3.4 (Contextual Genomics, Canada), a proprietary hypersensitive cancer hotspot assay which covers over 120 hotspots and 17 exons spanning 33 genes (Supplementary Table S3)[23]. Libraries were constructed using 75ng of total DNA input. Candidate somatic variants for orthogonal validation were selected from defined cancer hotspots reported in the Catalogue of Somatic Mutations in Cancer (COSMIC)[24], with probability scores $\geq 0.8$ (indicating the likelihood that a variant belongs to the mutation class as opposed to the artifact class) and variant allele frequency (VAF) $\geq 0.8 \%$.

\section{Orthogonal Validation via ddPCR}

Droplet digital polymerase-chain-reaction (ddPCR) was used to orthogonally validate hotspot mutations. DNA from each specimen was pre-amplified for 10 cycles before subsequent droplet generation using the QX200 Droplet Generator (Bio-Rad Laboratories, USA)[25]. In most cases, the same aliquot of extracted DNA used for targeted sequencing was also used for ddPCR validation (Supplementary Table S5 and S6). After thermal cycling, the QX200 Droplet Reader (Bio-Rad Laboratories, USA) was used to quantify droplets. See Supplementary Table S7 for a list of primers used and Supplementary Table S8 for ddPCR assay conditions.

\section{PTEN and ARIDIA Immunohistochemistry}

Loss of PTEN immunoreactivity was used as a surrogate for PTEN loss-of-function mutations. Following the protocol outlined in our previous study[10], specimens were stained on the 
Oncogenic Mutations in Eutopic Endometrium

Ventana Discovery Ultra (Ventana Medical Systems, USA) immunostainer using a 1:25 dilution of rabbit monoclonal antibody, 138G6 (Cell Signaling, USA). Similarly, loss of nuclear ARID1A immunoreactivity was used as a surrogate for ARID1A loss-of-function mutations[10,26,27]. Specimens were stained on the Dako Omnis (Agilent Technologies, USA) automated immunostainer using a 1:2000 dilution of ARID1 A rabbit monoclonal antibody EPR13501 (ab182560, Abcam, USA). TMN and BT-C. scored all ARID1A and PTEN immunostained slides.

\section{Results:}

\section{Somatic Driver Mutation Frequencies in Normal Endometrium}

As shown in Figure 1, we found that 16 of 25 women (64\%) who underwent hysterectomy and 43 of 85 women $(51 \%)$ who underwent D\&C or endometrial biopsy harbored at least one somatic alteration within their endometrial tissue samplings. Given our sample sizes, the prevalence of these alterations (whether specifically or overall) did not significantly differ between Hx and Bx cohorts (Supplementary Table S9). The most common somatic alterations were hotspot KRAS and PIK3CA mutations (affecting 31 of 110 and 14 of 110 total patients respectively), and focal loss of PTEN expression (defined by absence of staining in a small cluster of glands among normally expressing glands) (30 of 110 total patients) (Supplementary Figure S1). No patients exhibited loss of ARID1A either focally or globally.

\section{Anatomical Distribution of Oncogenic Changes}

For each woman in the Hx cohort, we independently collected and sequenced DNA from two tissue blocks representing endometrial tissue from the anterior and posterior uterus within the same patient. Somatic hotspot mutations were either completely absent (15 of 25 patients) or dissimilar (9 of 25 patients) between anterior and posterior samplings (Supplementary Figure $\mathrm{S} 2$ ). A single patient (Hx_25) harbored a KRAS G12A mutation in both samplings, however anatomical distortion of the uterus in this patient resulted in an inability to determine the spatial distinction between the two blocks of endometrial tissue (Table 1).

\section{Driver Mutations Across Age}

Accumulation of somatic alteration with increasing age has previously been suggested even in the context of normal tissues[16-19]. To assess the relationship between age and presence of somatic cancer-driver alterations, we generated a logistic regression model based on the sequencing and IHC findings for the 25 women in the Hx cohort and 85 women in the Bx cohort (Figure 2). Based on our model, the likelihood of a woman harbouring a somatic alteration in her endometrial tissue increases by $5 \%$ per year $(\mathrm{OR}=1.05,95 \% \mathrm{CI}=1.00-1.10, \mathrm{p}=0.035$, Wald test). Moreover, a model incorporating both age and menstrual phase (proliferative versus secretory phase) indicates that the prevalence of mutation is independent of menstrual phase ( $\mathrm{p}=$ 0.6309, Wald test) (Supplementary Figure S3).

\section{Comparison of Normal Endometrium Findings with Endometrial Cancer}

We compared our data with endometrial cancer data from The Cancer Genome Atlas (TCGA)[28] to better interpret mutational findings within the normal endometrium. Because our analysis is restricted to the hotspot regions in the 33 genes covered by the FIND IT ${ }^{\mathrm{TM}}$ assay (Supplemental Table S3), we limited our comparison to oncogenic mutations within these regions for the TCGA dataset. For our normal endometrial tissue cohort, we pooled our data 
Oncogenic Mutations in Eutopic Endometrium

from both the Hx cohort $(n=25)$ and Bx cohort $(n=85)$. Overall, endometrial cancer samples more frequently harbored oncogenic mutations compared to the patients examined in our study (96\% vs 54\%, p < 0.0001, Student's t-test) (Figure 3A; Supplementary Table S10). Women with endometrial cancer were far more likely to harbor a somatic mutation at a given age (OR: 10.8, 95\% CI: 3.83 - 33.3, p = 1.5 e-05, Wald test) (Supplementary Figure S4).

Gene-specific analysis mutation frequencies were either indistinguishable or higher in cancer specimens, except for KRAS driver mutations. We found a distinct trend ( $\mathrm{p}=0.0728)$ suggesting a greater proportion of non-cancer patients harbored $K R A S$ driver mutations in their endometrial tissue, 31 of 110 (28\%), compared with endometrial cancer patients, 48 of 248 (19\%) (Figure 3A; Supplementary Table S10). Indeed, in a model that incorporates age and KRAS mutation, whilst mutation rates in normal samples clearly increase with age $(\mathrm{OR}=1.06,95 \% \mathrm{CI}=1.01-$ $1.11, \mathrm{p}=0.01953$, Wald test), in the cancer samples the opposite trend is seen (OR $=0.96,95 \%$ $\mathrm{CI}=0.94-0.99, \mathrm{p}=0.0164$, Wald test) (Figure 3B).

\section{Discussion:}

In many cancers, tumor progression is largely dependent on the accumulation of so-called "driver mutations" that confer selective growth advantages on affected subclones compared to surrounding cells[29-31]. EAOCs, in fact are characterized by mutations affecting PIK3CA, PPP2R1A, ARIDIA, KRAS, CTNNB1, and PTEN[32]. Sporadic endometrial cancers also have a similar driver mutation landscape characterized by mutations affecting $P I K 3 C A, P I K 3 R I$, ARID1A, KRAS, CTNNB1, PTEN, FGFR2, and alterations in mismatch repair proteins[33]. The eutopic endometrium, an extremely dynamic tissue over the course of a woman's reproductive years, is both the tissue of origin for endometrial cancer and the presumed origin of EAOCs[6-8]. Therefore, it serves as an important reference for the studies of these cancers as well as endometriosis. In the current study, we have described (with orthogonal validation) the presence of somatic, driver mutations within histologically unremarkable, eutopic endometrium of over half of women lacking evidence of gynecologic malignancy.

Our findings are consistent with oncogenic mutations existing in small clonal pockets throughout the endometrium. Amongst informative patients, oncogenic mutations were dissimilar between the anterior and posterior samplings (i.e. if a particular point mutation were found in one sampling, it was not detected in the other sampling) - these findings were confirmed by ddPCR testing of both samplings for any mutation found in either block (Table 1; Supplementary Table S5). The VAFs of reported somatic mutations were generally low (between $0.8-11.9 \%$ in hysterectomy or D\&C/biopsy cases - see Table 1; Supplementary Table S5, S6), despite some enrichment of the glandular epithelial layer. This suggests that oncogenic mutations may be gland-level restricted, affecting only single (or a small number of) endometrial glands across a breadth of many glands from a given tissue specimen. This hypothesis seems well supported by two recent studies wherein individual endometrial glands were laser-captured from different areas within the endometrium of the same patient. Both studies suggest that different glands harbored different mutations[34,35]. These studies and our own results are also consistent with the findings by Mutter et al. (2014)[20],where PTEN-loss appears zonally in single, or small clusters of, glands (Supplementary Figure S1). Therefore, although our study represents broader sampling of the endometrium and mutations, our observations and those of others[34,35] are consistent with driver mutations, such as KRAS G12 mutations, occurring in small, clonal 
Oncogenic Mutations in Eutopic Endometrium

pockets throughout the uterus. Furthermore, it seems likely that acquisition of these mutations provides sufficient selective advantages to expand within an entire gland of affected endometrial cells, allowing this population to be detectable by our methods.

Somatic mutations have been previously described to accumulate with age in a variety of noncancerous tissues $[18,19,36]$ and thus we sought to determine the relationship between age and prevalence of somatic mutation in the normal endometrium. Overall, we found that the prevalence of oncogenic mutations in normal endometrium indeed increases by age; the risk of harboring such a mutation increases by approximately $5 \%$ per year. Moreover, the influence of age on mutation prevalence is independent of the phase of the menstrual cycle (Supplementary Figure S3). Comparing our data to the TCGA endometrial cancer dataset reveals that the prevalence of driver mutations is higher across age in endometrial cancer (or comparably as uncommon) in all genes except for KRAS. In our study, KRAS driver mutations occurred in over one-quarter of eutopic endometrium cases, illustrating a trend to greater prevalence in noncancerous vs endometrial cancer samples. Although the KRAS mutation rates in normal endometrial samplings increased with age the opposite was seen in the TCGA cancer samples. The latter observation is likely due to distinct $K R A S$ mutation rates in endometrial cancer subtypes that, themselves, have distinct age distributions[28]. Since patients in our study underwent hysterectomies, D\&Cs, or endometrial biopsies for benign uterine-related pathologies or related concerns, it is plausible that KRAS mutations may play a role in such benign/noncancer pathologies such as those described in arteriovenous malformations of the brain[37] or as we have previously observed in endometriosis[9,10]. It is also possible that early activation of $K R A S$ induces senescence[38] in endometrial cells, thereby inhibiting progression towards malignant transformation. In contrast to our high frequency KRAS mutations, CTNNB1 and ARIDIA are commonly mutated in endometrial cancer and EAOCs[32,33] but were not observed in any of our normal endometrium specimens. This may suggest that, within the context of endometrial-derived carcinomas, such mutations are later events in tumorigenesis or that accumulation of mutations in cancer-destined precursors is etiologically distinct from age-related accumulations.

Lastly, it is important to note that many of the cancer specimens in the TCGA dataset harbor multiple driver mutations within the same tumor. Although some of our normal endometrium specimens harbored multiple driver alterations within the same block, this was relatively uncommon and it remains unclear if mutations affected overlapping populations or independent subclones. The recent study by Moore et al. found two or more driver mutations in single endometrial glands in $22 \%$ of specimens[35], suggesting an elevated probability, albeit still nonconclusive, that driver mutations may co-exist in the same non-cancerous cells.

Within the eutopic endometrium, detection of driver mutations increases with age despite the lack of remarkable histological changes. Other factors, particularly oral contraceptive use or hormonal intrauterine device use may further modulate (reduce) risk of accumulating driver mutations within endometrial tissues, such as has been observed as a decline in the frequency of PTEN-null glands in women using these contraceptive methods[21] . Because of the lack of follow-up with patients in our study, it remains unclear what role, if any, these mutations play in the eutopic endometrium. Other obvious questions include whether mutations persist, harbored in early progenitor populations, or do they disappear at each menstrual cycle re-emerging de- 
Oncogenic Mutations in Eutopic Endometrium

novo with the rapid proliferation of the endometrial lining each month[20]? Our observation of surprisingly high frequency of driver mutations in the eutopic endometrium of women without evidence of malignancy or even subtle pathology suggests that care must be taken when interpreting detected mutations in non-cancer affected individuals. Ongoing preclinical development of mutation detection assays have suggested non-cancer affected populations are unaffected by somatic driver alterations[39], many of which overlapped with our assays. However, closer inspection suggests at least some studies may not show a full picture with both sampling methodology and control population age-bias potentially contributing to the failure to detect driver mutations in non-cancer populations. The development of screening methods for occult or pre-cancerous disease will need to incorporate appropriate specimen sampling, ageappropriate control populations, and a solid understanding of normal age-related somatic mutation accumulation.

In summary, our findings highlight the complexity of the eutopic endometrium and challenge our current understanding of so-called "normal tissues". As the mutations identified are obviously oncogenic in other tissues, these findings highlight the need to consider cell context and microenvironment along with mutation in the development of cancer. 
Oncogenic Mutations in Eutopic Endometrium

\section{Acknowledgements:}

This research is funded by the Canadian Cancer Society (Impact grant \#701603), Canadian Institutes of Health Research (Foundation grant \#154290), and the Janet D. Cottrelle Foundation through the BC Cancer Foundation. The VGH \& UBC Hospital Foundation and the BC Cancer Foundation provided funding to OVCARE: BC's Ovarian Cancer Research Team. A Frederick Banting and Charles Best Canada Graduate Scholarship from the Canadian Institutes of Health Research provided support to V. Lac. The Janet D. Cottrelle Foundation Scholars fund provided support to M.S. Anglesio. The Dr. Chew Wei Memorial Professorship in Gynecologic Oncology and the Canada Research Chairs program (Research Chair in Molecular and Genomic Pathology) provided support to D.G. Huntsman. The Michael Smith Foundation for Health Research Health Professional-Investigator Award provided support to P.J. Yong.

We would also like to acknowledge the staff at the Genetic Pathology Evaluation Centre (GPEC), the Department of Anatomical Pathology at the Vancouver General Hospital in Vancouver, OVCARE's gynaecological tissue bank, and the Calgary Laboratory Services for their assistance with specimen collection, immunohistochemical staining and optimization, and tissue sectioning.

\section{Statement of Author Contributions:}

VL, MSA, and DGH designed the study. TMN and BT-C identified specimens for the study, VL, AL, JK, TP, and MM processed samples, and performed experiments. TMN, BT-C, and MK reviewed pathology and generated/reviewed IHC data. VL and RA-H performed data analysis. AA and DC provided statistical design input and oversight. PJY and BCG provided clinical input to the study design. VL drafted the manuscript. All authors revised the manuscript and approved submission of the final version. 
bioRxiv preprint doi: https://doi.org/10.1101/561050; this version posted February 26, 2019. The copyright holder for this preprint (which was not certified by peer review) is the author/funder, who has granted bioRxiv a license to display the preprint in perpetuity. It is made available under aCC-BY-NC-ND 4.0 International license.

Oncogenic Mutations in Eutopic Endometrium

\section{References:}

1. Vercellini P, Vigano P, Somigliana E, et al. Endometriosis: pathogenesis and treatment. Nat Rev Endocrinol 2014;10:261-275.

2. Giudice LC. Clinical practice. Endometriosis. N Engl J Med 2010;362:2389-2398.

3. Sampson JA. Endometrial carcinoma of the ovary arising in endometrial tissue in that organ. American Journal of Obstetrics \& Gynecology 1925;9:111-114.

4. Pearce CL, Templeman C, Rossing MA, et al. Association between endometriosis and risk of histological subtypes of ovarian cancer: a pooled analysis of case-control studies. Lancet Oncol 2012;13:385-394.

5. Saavalainen L, Lassus H, But A, et al. Risk of Gynecologic Cancer According to the Type of Endometriosis. Obstet Gynecol 2018;131:1095-1102.

6. Sato N, Tsunoda H, Nishida M, et al. Loss of heterozygosity on 10q23.3 and mutation of the tumor suppressor gene PTEN in benign endometrial cyst of the ovary: possible sequence progression from benign endometrial cyst to endometrioid carcinoma and clear cell carcinoma of the ovary. Cancer Res 2000;60:7052-7056.

7. Wiegand KC, Shah SP, Al-Agha OM, et al. ARID1A mutations in endometriosis-associated ovarian carcinomas. N Engl J Med 2010;363:1532-1543.

8. Anglesio MS, Bashashati A, Wang YK, et al. Multifocal endometriotic lesions associated with cancer are clonal and carry a high mutation burden. J Pathol 2015;236:201-209.

9. Anglesio MS, Papadopoulos N, Ayhan A, et al. Cancer-Associated Mutations in

Endometriosis without Cancer. N Engl J Med 2017;376:1835-1848.

10. Lac V, Verhoef L, Aguirre-Hernandez R, et al. Iatrogenic endometriosis harbors somatic cancer-driver mutations. Hum Reprod 2019;34:69-78.

11. Burney RO, Giudice LC. Pathogenesis and pathophysiology of endometriosis. Fertil Steril 2012;98:511-519.

12. Sanfilippo JS, Wakim NG, Schikler KN, et al. Endometriosis in association with uterine anomaly. Am J Obstet Gynecol 1986;154:39-43.

13. D'Hooghe TM, Bambra CS, Raeymaekers BM, et al. Intrapelvic injection of menstrual endometrium causes endometriosis in baboons (Papio cynocephalus and Papio anubis). Am J Obstet Gynecol 1995;173:125-134.

14. D'Hooghe TM, Bambra CS, Suleman MA, et al. Development of a model of retrograde menstruation in baboons (Papio anubis). Fertil Steril 1994;62:635-638.

15. Masuda H, Matsuzaki Y, Hiratsu E, et al. Stem cell-like properties of the endometrial side population: implication in endometrial regeneration. PLoS One 2010;5:e10387.

16. Martincorena I, Roshan A, Gerstung M, et al. Tumor evolution. High burden and pervasive positive selection of somatic mutations in normal human skin. Science 2015;348:880-886.

17. Jacobs KB, Yeager M, Zhou W, et al. Detectable clonal mosaicism and its relationship to aging and cancer. Nat Genet 2012;44:651-658.

18. Krimmel JD, Schmitt MW, Harrell MI, et al. Ultra-deep sequencing detects ovarian cancer cells in peritoneal fluid and reveals somatic TP53 mutations in noncancerous tissues. Proc Natl Acad Sci U S A 2016;113:6005-6010.

19. Martincorena I, Fowler JC, Wabik A, et al. Somatic mutant clones colonize the human esophagus with age. Science 2018;362:911-917.

20. Mutter GL, Monte NM, Neuberg D, et al. Emergence, involution, and progression to carcinoma of mutant clones in normal endometrial tissues. Cancer Res 2014;74:2796-2802. 
Oncogenic Mutations in Eutopic Endometrium

21. Lin MC, Burkholder KA, Viswanathan AN, et al. Involution of latent endometrial precancers by hormonal and nonhormonal mechanisms. Cancer 2009;115:2111-2118.

22. Monte NM, Webster KA, Neuberg D, et al. Joint loss of PAX2 and PTEN expression in endometrial precancers and cancer. Cancer Res 2010;70:6225-6232.

23. Aparicio S, Shah S, Aguirre-Hernandez R, et al. MOLECULAR QUALITY ASSURANCE METHODS FOR USE IN SEQUENCING. 2018;.

24. Forbes SA, Beare D, Boutselakis H, et al. COSMIC: somatic cancer genetics at highresolution. Nucleic Acids Res 2017;45:D783.

25. Lac V, Huntsman DG. Distinct developmental trajectories of endometriotic epithelium and stroma: implications for the origins of endometriosis. J Pathol 2018;

26. Khalique S, Naidoo K, Attygalle AD, et al. Optimised ARID1A immunohistochemistry is an accurate predictor of ARID1A mutational status in gynaecological cancers. J Pathol Clin Res 2018;

27. Kobel M, Anglesio MS, Brenton JD. You won't believe this old test ... that does cheap single-cell mutation detection. J Pathol Clin Res 2018;

28. Cancer Genome Atlas Research Network, Kandoth C, Schultz N, et al. Integrated genomic characterization of endometrial carcinoma. Nature 2013;497:67-73.

29. Hanahan D, Weinberg RA. The hallmarks of cancer. Cell 2000;100:57-70.

30. Hanahan D, Weinberg RA. Hallmarks of cancer: the next generation. Cell 2011;144:646674.

31. Stratton MR, Campbell PJ, Futreal PA. The cancer genome. Nature 2009;458:719-724. 32. Worley MJ, Welch WR, Berkowitz RS, et al. Endometriosis-associated ovarian cancer: a review of pathogenesis. Int J Mol Sci 2013;14:5367-5379.

33. O'Hara AJ, Bell DW. The genomics and genetics of endometrial cancer. Adv Genomics Genet 2012;2012:33-47.

34. Suda K, Nakaoka H, Yoshihara K, et al. Clonal Expansion and Diversification of CancerAssociated Mutations in Endometriosis and Normal Endometrium. Cell Rep 2018;24:1777-1789. 35. Moore L, Leongamornlert D, Coorens TH, et al. The mutational landscape of normal human endometrial epithelium. bioRxiv 2018;.

36. Risques RA, Kennedy SR. Aging and the rise of somatic cancer-associated mutations in normal tissues. PLoS Genet 2018;14:e1007108.

37. Nikolaev SI, Vetiska S, Bonilla X, et al. Somatic Activating KRAS Mutations in Arteriovenous Malformations of the Brain. N Engl J Med 2018;378:250-261.

38. Robinson G. The interrelationship of anxiety, hostility and guilt; some physiological correlations. J Asthma Res 1967;5:115-121.

39. Wang Y, Li L, Douville C, et al. Evaluation of liquid from the Papanicolaou test and other liquid biopsies for the detection of endometrial and ovarian cancers. Sci Transl Med 2018;10:10.1126/scitranslmed.aap8793. 
Oncogenic Mutations in Eutopic Endometrium

Table 1. Mutational findings within normal endometrium samples from hysterectomy patients and corresponding variant allele frequencies (VAF) determine through targeted sequencing and droplet digital PCR (ddPCR).

\begin{tabular}{|c|c|c|c|c|c|}
\hline $\begin{array}{l}\text { Patient and } \\
\text { Block ID }\end{array}$ & $\begin{array}{l}\text { Region of Uterus } \\
\text { Sampled }\end{array}$ & PTEN IHC & $\begin{array}{l}\text { Driver Mutation } \\
\text { Identified }\end{array}$ & $\begin{array}{l}\text { VAF (\%) - } \\
\text { Sequencing }\end{array}$ & $\begin{array}{l}\text { VAF (\%) - } \\
\text { ddPCR }\end{array}$ \\
\hline$H x \_2 A$ & anterior & normal & & & \\
\hline $\mathrm{Hx} \_2 \mathrm{~B}$ & posterior & normal & ERBB2 S310F & 0.974 & 0.792 \\
\hline $\mathrm{Hx} \_4 \mathrm{~A}$ & anterior & loss - zonal & & & \\
\hline $\mathrm{Hx} \_4 \mathrm{~B}$ & posterior & normal & & & \\
\hline $\mathrm{Hx} \_5 \mathrm{~A}$ & anterior & loss - zonal & & & \\
\hline $\mathrm{Hx} \_5 \mathrm{~B}$ & posterior & normal & & & \\
\hline$H x \_6 A$ & anterior & normal & KRAS G12V & 2.654 & 1.63 \\
\hline$H x \_6 B$ & posterior & normal & KRAS G12A & 1.408 & 1.08 \\
\hline $\mathrm{Hx} \_7 \mathrm{~A}$ & posterior & loss - zonal & & & \\
\hline $\mathrm{Hx} \_7 \mathrm{~B}$ & anterior & normal & & & \\
\hline $\mathrm{Hx} \_8 \mathrm{~A}$ & posterior & loss - zonal & & & \\
\hline $\mathrm{Hx} \_8 \mathrm{~B}$ & anterior & loss - zonal & & & \\
\hline Hx_9A & anterior & normal & & & \\
\hline $\mathrm{Hx} \_9 \mathrm{~B}$ & posterior & loss - zonal & KRAS G13D & 2.16 & 1.95 \\
\hline$H x \_10 A$ & $\mathrm{n} / \mathrm{a}$ & normal & & & \\
\hline Hx_10B & $\mathrm{n} / \mathrm{a}$ & loss - zonal & & & \\
\hline$H x \_12 A$ & anterior & normal & PIK3CA R88Q & 1.337 & 1.23 \\
\hline$H x \_12 B$ & posterior & normal & & & - \\
\hline \multirow[t]{2}{*}{$H x \_13 A$} & \multirow[t]{2}{*}{ posterior } & \multirow[t]{2}{*}{ normal } & PIK3CA H1047R & 2.497 & 3.36 \\
\hline & & & KRAS G12C & 1.036 & 0.538 \\
\hline$H x \_13 B$ & anterior & normal & & & \\
\hline \multirow[t]{2}{*}{$H x \_16 A$} & \multirow[t]{2}{*}{ anterior } & \multirow[t]{2}{*}{ normal } & KRAS G12V & 1.079 & 1.06 \\
\hline & & & PIK3CA E542K & 1.015 & 0.897 \\
\hline$H x \_16 B$ & posterior & loss - zonal & PIK3CA H1047R & 2.402 & 1.92 \\
\hline \multirow[t]{2}{*}{$\mathrm{Hx} \_18 \mathrm{~A}$} & \multirow[t]{2}{*}{ anterior } & \multirow[t]{2}{*}{ normal } & FGFR2 K659E & 5.505 & 7.77 \\
\hline & & & KRAS G12V & 1.551 & 1.24 \\
\hline $\mathrm{Hx} \_18 \mathrm{~B}$ & posterior & loss - zonal & & & \\
\hline \multirow[t]{2}{*}{$H x \_21 \mathrm{~A}$} & \multirow[t]{2}{*}{ anterior } & \multirow[t]{2}{*}{ normal } & KRAS G12D & 5.587 & 4.71 \\
\hline & & & PIK3CA M1043I & 2.39 & 2.27 \\
\hline \multirow[t]{3}{*}{$H x \_21 B$} & \multirow[t]{3}{*}{ posterior } & \multirow[t]{3}{*}{ normal } & KRAS G12V & 6.112 & 6.32 \\
\hline & & & PIK3CA H1047R & 2.373 & 2.76 \\
\hline & & & FGFR2 S252W & 1.889 & 1.17 \\
\hline$H x \_22 A$ & anterior & loss - zonal & & & \\
\hline$H x \_22 B$ & posterior & normal & & & \\
\hline$H x \_23 A$ & anterior & $\mathrm{n} / \mathrm{a}$ & & & \\
\hline$H x \_23 B$ & posterior & normal & KRAS G12D & 11.866 & 10.2 \\
\hline$H x \_25 \mathrm{~A}$ & $\mathrm{n} / \mathrm{a}$ & normal & KRAS G12A & 1.584 & 1.60 \\
\hline Hx_25B & $\mathrm{n} / \mathrm{a}$ & loss - zonal & KRAS G12A & 1.670 & 1.00 \\
\hline
\end{tabular}

*Data for nine patients without detectable somatic mutations or abnormal immunohistochemistry findings are omitted from this table. 
Oncogenic Mutations in Eutopic Endometrium

\section{Figure Legends:}

Figure 1. Overview of somatic, oncogenic alterations detected in the eutopic endometrium from (A) hysterectomy patients or (B) dilation and curettage or endometrial biopsy patients. Red rectangles denote driver mutations detected by targeted sequencing, whereas blue rectangles denote (zonal) loss detected through immunohistochemistry studies. The asterisk $(*)$ denotes a patient wherein zonal loss of PTEN as well as a PTEN driver mutation was detected.

Figure 2. The prevalence of somatic driver mutations in the eutopic endometrium across age. According to this logistic regression model, the prevalence of mutation increases with age (OR: 1.05 (95\% CI: $1.00-1.10), \mathrm{p}=0.035$, Wald test).

Figure 3. (A) Comparison of the prevalence of somatic mutations in endometrial cancer versus histologically normal eutopic endometrium (mutations found in endometrial cancer but were not detected in any eutopic endometrium sample are not displayed). (B) KRAS mutation prevalence across age in endometrial cancer samples versus normal endometrium samples. In normal endometrium samples, the prevalence of $K R A S$ mutation increases with age $(\mathrm{OR}=1.06,95 \% \mathrm{CI}$ $=1.01-1.11, \mathrm{p}=0.01953$, Wald test $)$, whereas it decreases with age in endometrial cancer samples $(\mathrm{OR}=0.96,95 \% \mathrm{CI}=0.94-0.99, \mathrm{p}=0.0164$, Wald test $)$.

\section{Supplementary Figure Legends:}

Supplementary Figure S1. PTEN IHC studies of eutopic endometrium specimens showing zonal loss of PTEN in epithelial cells and matching H\&E staining.

Supplementary Figure S2. Comparison of point mutations detected in anterior and posterior samplings from patients in the Hx cohort.

Supplementary Figure S3. Logistic regression model of mutation prevalence across age in secretory phase versus proliferative phase endometrium

Supplementary Figure S4. Logistic regression model of mutation prevalence across age in endometrial cancer versus normal endometrium. 


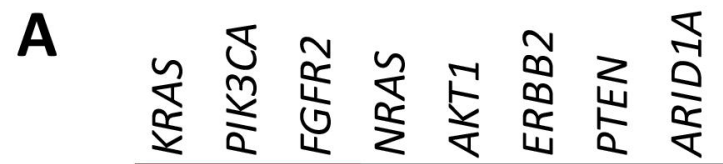

$H x \_21$
$H x \_16$

$\mathrm{Hx} \_13$
$\mathrm{Hx} \_18$

$\mathrm{Hx} 09$

Hx_25

Hx_06

Hx_23

$\mathrm{Hx} \_12$

$\mathrm{Hx} 02$

Hx_04

Hx_05

$\mathrm{Hx} \_07$

Hx_08

$\mathrm{Hx} 10$

Hx_22

$\mathrm{Hx} \_01$

$\mathrm{Hx} 03$

$\mathrm{Hx} \_11$

Hx_14

$\mathrm{Hx}-15$

$\mathrm{Hx} \_17$

$\mathrm{Hx} \_19$

Hx_20

Hx_24
B

Bx_012

Bx 053

Bx_021

Bx_009

Bx_061

Bx 068

Bx_071

Bx_101

Bx_003

Bx_006

Bx 015

Bx_018

Bx_022

Bx_037

Bx_040

Bx 052

Bx_060

Bx_064

Bx 065

Bx_067

Bx_099

Bx_046

Bx_082

Bx 016

Bx_005

Bx 051

Bx_084

$\mathrm{Bx} \_100$

Bx 008

Bx_023

Bx 010

Bx_026

Bx_034

Bx 043

Bx_045

Bx 049

Bx_050

Bx_054

Bx 074

Bx_076

Bx_079

Bx_001

Bx_002

Bx 004

Bx_007

Bx_011

Bx 014

Bx_017

Bx 019

Bx_020

$\mathrm{Bx} \_024$

Bx 025

Bx_027

Bx_028

Bx_029

Bx_030

Bx_031

Bx_032

Bx_033

Bx_035

Bx_036

Bx 038

Bx_039

Bx_041

Bx_042

Bx_044

Bx 047

Bx_048

Bx_055

Bx_057

Bx_058

Bx 059

Bx_062

Bx_063

Bx_066

Bx_069

Bx 072

Bx_073

Bx_080

Bx 081

Bx_083

Bx_093

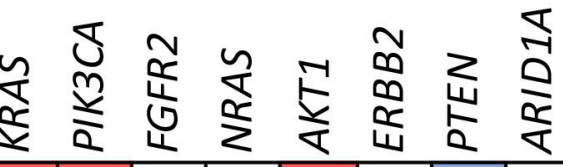

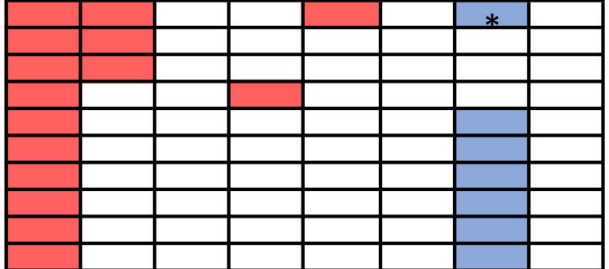

\begin{tabular}{|l|l|l|l|l|l|l|}
\hline \\
\hline
\end{tabular}

\begin{tabular}{|l|l|l|l|l|l|l|l|}
\hline & & & & & & & \\
\hline & & & & & & & \\
\hline & & & & & & & \\
\hline
\end{tabular}

\begin{tabular}{|l|l|l|l|l|l|l|l|}
\hline & & & & & & & \\
\hline & & & & & & & \\
\hline
\end{tabular}

\begin{tabular}{|l|l|l|l|l|l|l|l|}
\hline & & & & & & & \\
\hline & & & & & & & \\
\hline
\end{tabular}

\begin{tabular}{|l|l|l|l|l|l|l|l|}
\hline & & & & & & & \\
\hline & & & & & & & \\
\hline
\end{tabular}

\begin{tabular}{|l|l|l|l|l|l|l|}
\hline & & & & & & \\
\hline
\end{tabular}

\begin{tabular}{|l|l|l|l|l|l|l|l|}
\hline \\
\hline
\end{tabular}

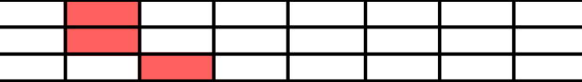

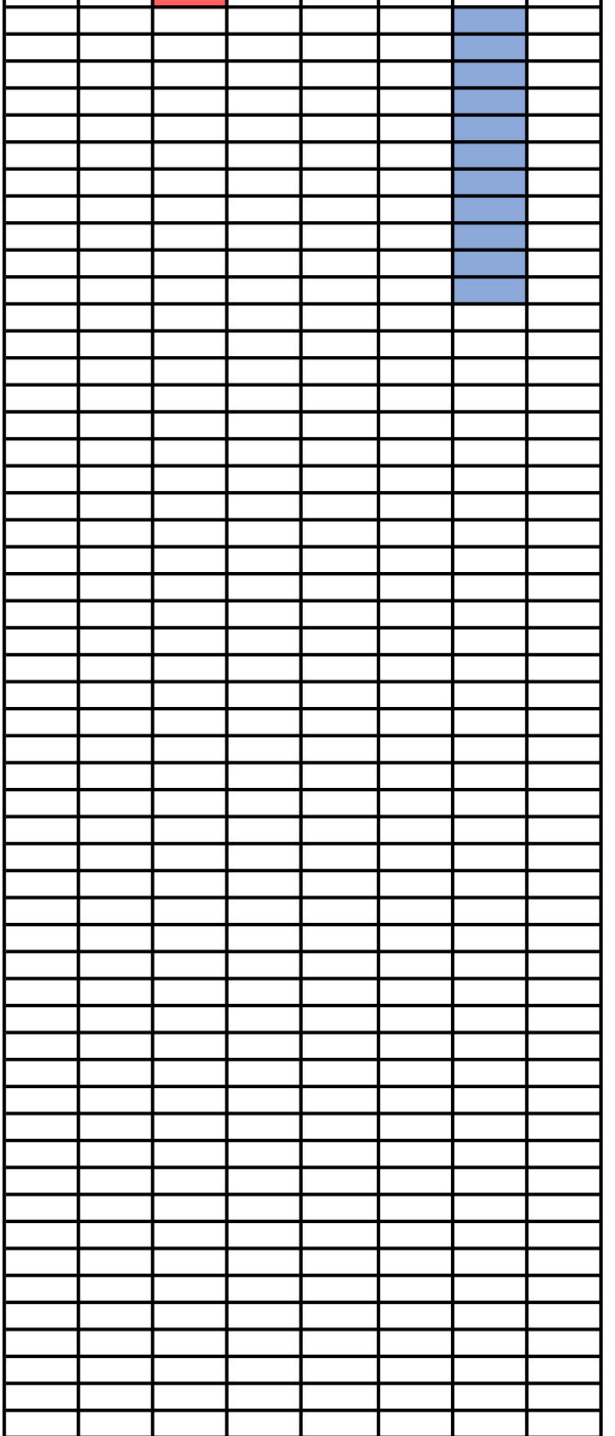



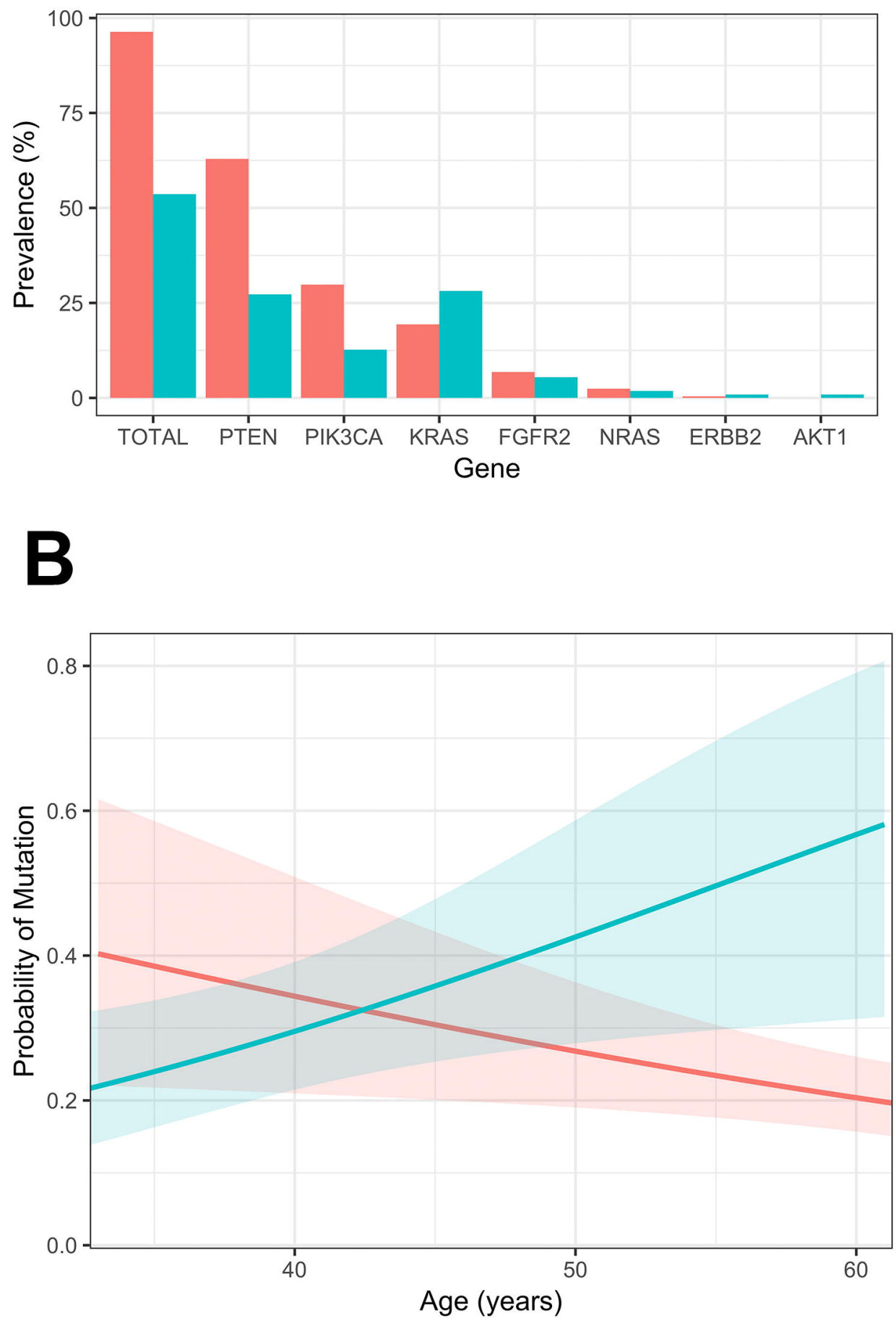

Cohort

Endometrial Cancer Normal Endometrium

\section{Cohort}

- endometrial_cancer

— normal_endo 


\section{PTEN \\ H\&E}

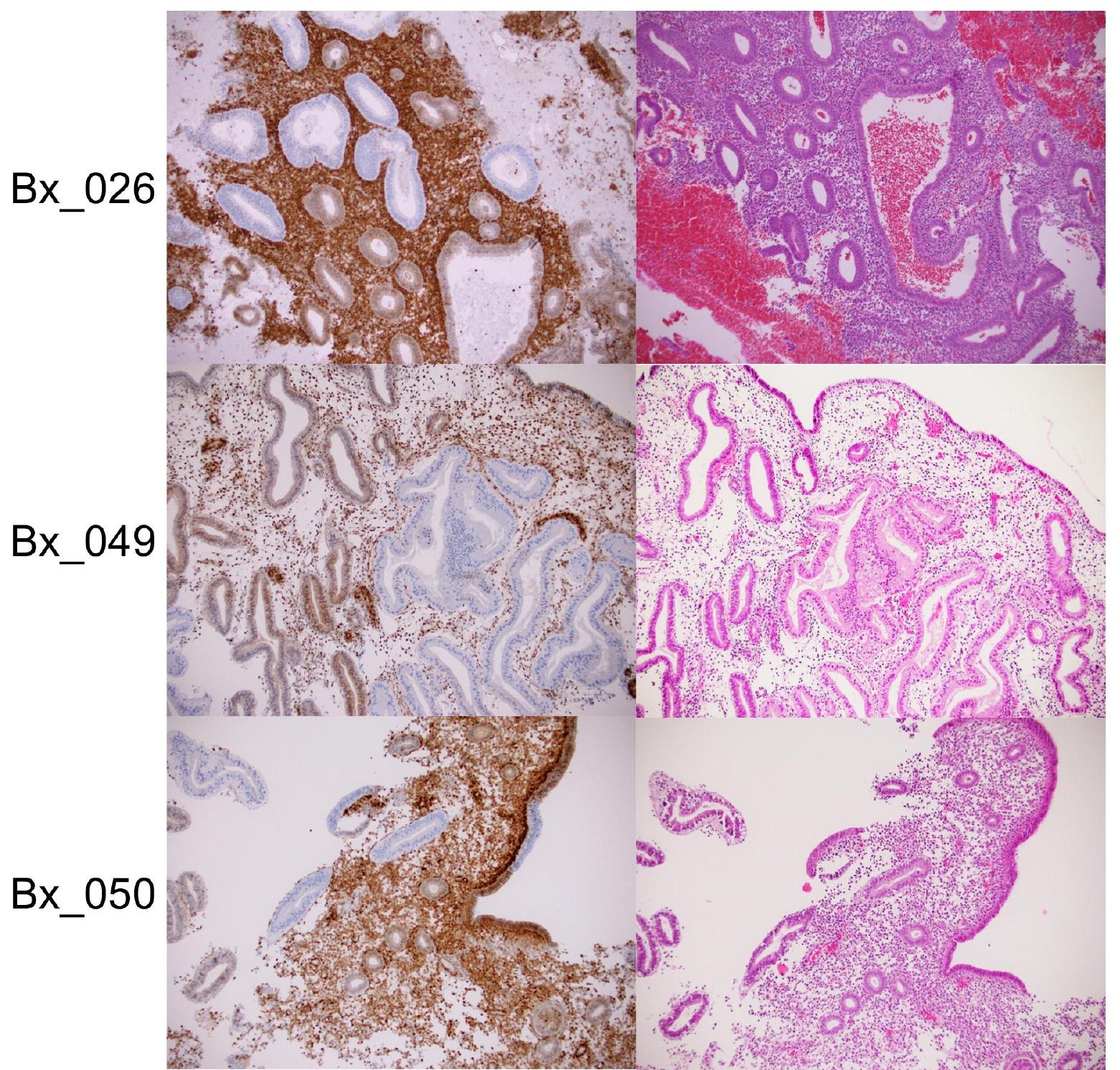




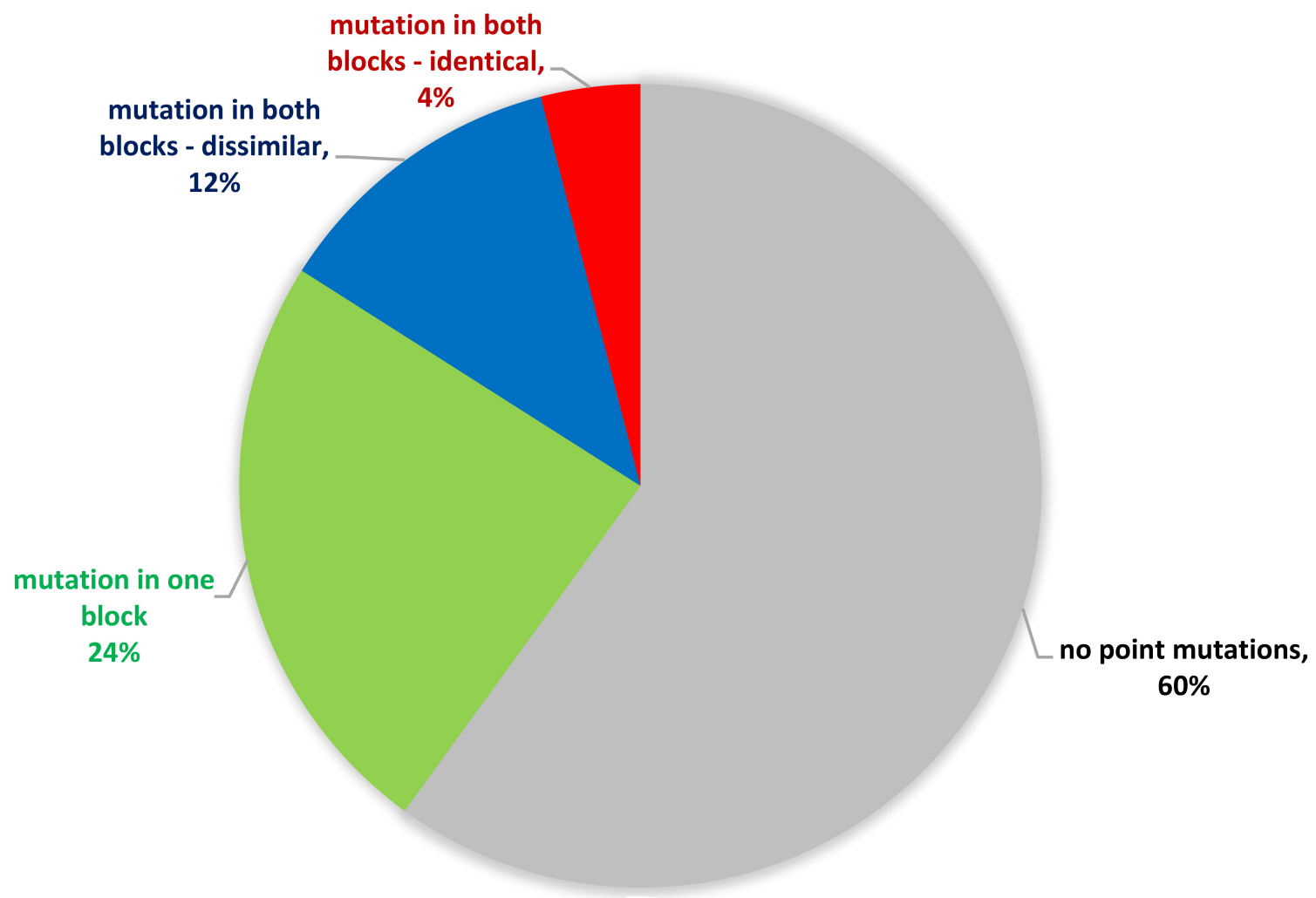


